\title{
Clinical relevance of standardized mobile gait tests - reliability analysis between gait recordings at hospital and home in Parkinson's disease: A pilot study
}

Heiko Gaßner ${ }^{*}$, Philipp Sanders ${ }^{1}$, Alisa Dietrich ${ }^{1}$, Franz Marxreiter ${ }^{1}$, Bjoern M. Eskofier $^{2}$, Jürgen Winkler ${ }^{1}$, Jochen Klucken ${ }^{1} 34$

${ }^{1}$ Department of Molecular Neurology, University Hospital Erlangen, Friedrich-Alexander University Erlangen-Nürnberg (FAU), Erlangen, Germany,

2 Machine Learning and Data Analytics Lab, Friedrich-Alexander University Erlangen-Nürnberg (FAU), Erlangen, Germany

${ }^{3}$ Medical Valley - Digital Health Application Center GmbH, Bamberg, Germany

${ }^{4}$ Fraunhofer Institute for Integrated Circuits IIS, Erlangen, Germany

\section{Corresponding author}

*Heiko Gaßner, PhD

Gait analysis specialist

Department of Molecular Neurology

University Hospital Erlangen

Schwabachanlage 6

91054 Erlangen, Germany

Word count Abstract

Word count Manuscript

Key Words: Parkinson's disease, Gait analysis, Wearable sensors, Telemedicine, Home Monitoring

The final publication is available at IOS Press through http://dx.doi.org/10.3233/JPD-202129 


\section{Acknowledgment}

The authors would like to thank all patients that participated in this study. The study was supported by the Bavarian Ministry of Economic Affairs, Regional Development and Energy, and performed at the Department of Molecular Neurology (University Hospital Erlangen, Erlangen, Germany). The present work is part of the doctoral thesis of Philipp Sanders in the frame of obtaining the degree „Dr. med.” at the Medical Faculty, Friedrich-Alexander University Erlangen-Nürnberg (FAU). Heiko Gaßner, Bjoern Eskofier, and Jochen Klucken are supported by Mobilise-D from the Innovative Medicines Initiative 2 Joint Undertaking under grant agreement no. 820820. We further received support by the Manfred Roth-Stiftung and Forschungsstiftung Medizin am Universitätsklinikum Erlangen as well as the Federal Ministry of Education and Research (project: treatHSP, grant number 01GM1905). Bjoern Eskofier gratefully acknowledges the support of the German Research Foundation (DFG) within the framework of the Heisenberg professorship programme (grant number ES 434/8-1). 


\section{ABSTRACT (250/250 words)}

Background: Gait impairments in Parkinson's disease (PD) are quantified using inertial sensors under standardized test settings in the hospital. Recent studies focused on the assessment of freeliving gait in PD. However, the clinical relevance of standardized gait tests recorded at the patient's home is unclear.

Objective: To evaluate the reliability of supervised, standardized sensor-based gait outcomes at home compared to the hospital.

Methods: Patients with PD $(n=20)$ were rated by a trained investigator using the Unified Parkinson Disease Rating Scale (UPDRS-III). Gait tests included a standardized 4x10 m walk test and the Timed Up and Go Test (TUG). Tests were performed in the hospital (HOSPITAL) and at patients' home (HOME), and controlled for investigator, time of the day, and medication. Statistics included reliability analysis using Intra-Class correlations and Bland-Altman plots.

Results: UPDRS-III and TUG were comparable between HOSPITAL and HOME. PD patients' gait at HOME was slower (gait velocity $\Delta=-0.07 \pm 0.11 \mathrm{~m} / \mathrm{s},-6.1 \%$ ), strides were shorter (stride length $\Delta=-9.2 \pm 9.4 \mathrm{~cm} ;-7.3 \%$ ), and shuffling of gait was more present (maximum toe-clearance $\Delta=-0.7 \pm 2.5 \mathrm{~cm} ;-8.8 \%)$. Particularly, narrow walkways $(<85 \mathrm{~cm})$ resulted in a significant reduction of gait velocity at home. Reliability analysis (HOSPITAL vs. HOME) revealed excellent ICC coefficients for UPDRS-III $(0.950, \mathrm{p}<0.000)$ and gait parameters (e.g. stride length: $0.898, \mathrm{p}<0.000$; gait velocity: 0.914,p<0.000; stance time: $0.922, \mathrm{p}<0.000$; stride time: $0.907, \mathrm{p}<0.000$ ).

Conclusions: This pilot study underlined the clinical relevance of gait parameters by showing excellent reliability for supervised, standardized gait tests at HOSPITAL and HOME, even though gait parameters were different between test conditions. 


\section{Introduction}

Gait in Parkinson's disease (PD) is highly affected by prototypical symptoms including bradykinesia, and rigidity reflected by reduced amplitude and automaticity of movement [1]. Gait impairment plays an important role upon motor functioning as it affects the quality of life, limits the independence of PD patients, and reduces activities of daily living [2, 3]. Objective gait parameters may provide metric, granular information complementing the Unified Parkinson Disease Rating Scale, part III (UPDRS-III) assessed by neurologists [4]. Moreover, inertial sensors have the potential to record additional information as comprehensive and long-lasting monitoring tool [5]. To reach this goal of implementing technologies into daily care of PD patients, the clinical relevance of sensor-based outcomes must be shown at the individual home situation. In this study, a first step will be done by comparing standardized gait assessments in the hospital and home environment.

\section{Quantification of gait impairment using wearable technologies}

In the emerging field of wearable technologies, inertial sensors (accelerometers and gyroscopes) combined with machine learning algorithms have shown to provide objective, metric outcomes that support the rather rater- and time-dependent clinical ratings of motor symptoms in chronic neurological diseases such as Parkinson's disease [4, 6-8], Multiple sclerosis [9] or Huntington's disease [10]. Using wearable technologies, clinicians have the opportunity to gain a comprehensive picture of motor impairment by determining deficits that cannot be observed by eye. Inertial sensors provide the potential advantage to measure clinically relevant data as motor symptoms, fall risk, freezing of gait, functional mobility or physical activity [11]. Wearable technologies may provide added value as they are able to quantify gait at home or in different community settings [1]. However, to date there is no fully validated system for monitoring clinical features in the home environment [11]. 
The long-term goal is to interpret free-living gait in order to improve therapy and quality of life of PD patients. However, there are numerous variables and conditions in free-living gait that need to be taken into account. Recording free-living gait data over 8 hours without any reference is very hard to interpret for a clinical meaningful purpose. Therefore, we intend to perform a step-by-step approach including these steps: starting from 1) standardized + supervised conditions in the hospital over 2) standardized + supervised assessments at home (presented in this study) to 3) standardized + unsupervised gait at home and finally 4) free-living gait. Herewith, we try to overcome the large step between assessments in the hospital and free-living gait in order to extract clinically relevant information.

\section{Inertial sensor-based gait analysis outside lab}

The assessment of gait in the patient's home (HOME) is an important, complementing approach in comparison to the clinical setting (HOSPITAL). Wearable technologies enable gait analysis not only in the hospital but also in variable, real-life environments like at patients' homes [12]. Inertial sensors have the potential to continuously record gait over several hours instead of generating snapshots of gait impairments during short-lasting visits in the outpatient unit of hospitals [13, 14]. These technical possibilities moved in the focus of recent studies to assess gait outside the clinical setting. As a currently published review pointed out, home-based free living gait recordings are of huge interest of research projects using inertial sensors [15]. Current studies dealing with gait analysis in the home environment evaluate which home-based gait measures provide relevant information with regard to disease-specific gait impairment. Morris et al. showed that conceptual gait domains (pace, rhythm, variability, asymmetry) defined for the at-lab setting in PD were congruent with free-living gait [16] indicating that home-based measures provide meaningful data. Del Din et al. observed that longer gait bouts ( $>10 \mathrm{~s})$ solely derived from free-living gait showed differences between $47 \mathrm{PD}$ patients and 50 controls with regard to pace, rhythm, and asymmetry indicating that especially longer gait bouts include clinically relevant information about gait 
characteristics [17]. However, free-living gait largely depends on context information and environmental factors which need to be captured in addition to sensor data and put into the complex picture of gait in everyday life. Otherwise, it is not possible to interpret free-living gait patterns correctly and derive clinically relevant information. Moving one step back, it is necessary to understand the impact of environmental factors first, before we interpret real-life gait.

Recent studies focused on the assessment of free-living gait in PD, however, the evaluation of gait parameters derived from identical supervised gait test in the hospital and at home (HOSPITAL vs. HOME) is still missing for PD. Moreover, the clinical value of standardized gait outcomes recorded at the patient's home are not clearly understood. Acknowledging that gait patterns are modified during gait tests in presence of an observer in comparison to unobserved situations [18], this study focused on the evaluation of standardized gait tests in patients' homes in order to understand the impact of home conditions on gait performance.

The aim of this pilot study was to evaluate the reliability of gait parameters gathered by standardized, supervised gait tests performed in hospital and home-environment in order to understand the clinical relevance of home-based measures.

We hypothesize that sensor-based gait parameters measured in standardized gait tests at hospital will be better and cannot be directly compared to the identical assessment in patients' homes. However, we expect that there is clinical meaningful information in gait data recorded at home.

\section{Subjects and Methods}

\section{Study cohort}

Twenty patients diagnosed with PD were enrolled at the movement disorder unit, Department of Molecular Neurology, University Hospital Erlangen, Germany. Sporadic PD was defined according to the Guidelines of the German Association for Neurology (DGN) similar to the UK PD Society Brain Bank criteria [19]. PD patients with an age $>18$ years and Hoehn and Yahr disease stage (H\&Y) between I-III were included. Exclusion criteria consisted of motor fluctuations, dyskinesia, 
and comorbidities potentially affecting gait (e.g. polyneuropathy, visual problems orthopedic, psychiatric comorbidities, orthostatic hypotensi

on). Participants received standardized clinical assessments including UPDRS-III, H\&Y and Montreal Cognitive Assessment (MoCA). Postural Instability and Gait Difficulty subscore (PIGD) was calculated as defined $[8,20]$ as sum of UPDRS-III items 'gait', 'postural stability', 'arising from chair', and 'posture'. Furthermore, the patients filled in questionnaires to evaluate their fear of falling (Falls Efficacy Scale-International, FES-I) and their falls history. Characteristics of the study population are presented in Table 1. This study was approved by the local ethics committee (reference number: 166_18 B, Medical Faculty, FAU Erlangen-Nürnberg, Germany), and participants gave written informed consent according to the Declaration of Helsinki.

\begin{tabular}{|c|c|c|c|c|}
\hline \multirow[b]{2}{*}{ Age, years } & \multicolumn{3}{|c|}{$\begin{array}{c}\text { PD Patients }(n=20) \\
\text { mean } \pm \text { SD }\end{array}$} & \multirow[t]{2}{*}{$\begin{array}{c}\text { Range } \\
\text { (Min - Max) }\end{array}$} \\
\hline & 72.6 & \pm & 7.9 & \\
\hline Gender, male / female & & 121 & & \\
\hline Height, cm & 174.2 & \pm & 8.8 & \\
\hline Weight, kg & 80.7 & \pm & 13.7 & \\
\hline $\mathrm{BMI}, \mathrm{kg} / \mathrm{m}^{2}$ & 26.6 & \pm & 4.4 & \\
\hline Disease duration, years & 7.15 & \pm & 4.3 & \\
\hline LEDD, mg/d & 599.5 & \pm & 327.2 & \\
\hline FES-I & 22.8 & \pm & 6.1 & \\
\hline Falls within last 12 months Yes / No & & +1 & & \\
\hline H\&Y stage & 2.4 & \pm & 0.4 & \\
\hline H\&Y 1.5-2, n & & 7 & & \\
\hline $\mathrm{H} \& Y 2.5-3, \mathrm{n}$ & & 13 & & \\
\hline UPDRS-III at hospital & 21.3 & \pm & 7.6 & $8-37$ \\
\hline PIGD & 2.5 & \pm & 1.8 & $0-7$ \\
\hline MoCA & 26.9 & \pm & 3.3 & $19-30(n=4<26)$ \\
\hline
\end{tabular}

Table 1: Characteristics of PD patients. LEDD: Levodopa equivalent daily dose, FES-I: Falls Efficacy Scale, H\&Y: Hoehn and Yahr disease stage, UPDRS-III: Motor score of the Unified Parkinson's disease rating scale, PIGD: Postural Instability and Gait Difficulty subscore, MoCA: Montreal Cognitive Assessment, Significance level p $<0.05$. 


\section{Study procedures}

PD patients performed a standardized $4 \times 10 \mathrm{~m}$ walk test using sensor-based gait analysis [21] and the Timed Up and Go Test (TUG) [22] supervised by an investigator. In a time frame of 7 days (mean, SD: $7.1 \pm 10.0$ days) after the assessment in the hospital (HOSPITAL) the clinical scoring (UPDRS-III) and sensor-based gait analysis were repeated at the patients' home environment (HOME) by the same trained investigator. The assessments in the HOME condition were performed at the same time of the day compared to the HOSPITAL. Participants were asked to take their medication on both testing days exactly at the identical time and dosage. In the hospital, participants performed a standardized $4 \times 10 \mathrm{~m}$ overground gait test on a $10 \mathrm{~m}$ long corridor in self-selected walking speed and without stops at turning points. In the home environment, PD patients repeated the same standardized and supervised test in self-selected speed on a walkway of maximal possible walking distance (4x maximum available distance) in the patients' rooms (mean, SD: $7.8 \pm 1.5 \mathrm{~m}$, range 6-10 m). Importantly, usually the distance included the transfer from room to room through a door. Only straight strides were automatically detected by the stride detection algorithm [21] and used for gait parameter calculations as described [23].

\section{Sensor-based gait analysis}

Gait characteristics were evaluated in a standardized $4 \times 10 \mathrm{~m}$ gait test using an instrumented, sensorbased gait analysis system. This system consists of inertial SHIMMER sensors (Shimmer Research Ltd., Dublin, Ireland) laterally attached to the posterior portion of both shoes [8]. Gait signals were recorded within a (tri-axial) accelerometer range of $\pm 6 \mathrm{~g}$ (sensitivity $300 \mathrm{mV} / \mathrm{g}$ ) and a gyroscope range of \pm 500 degree/sec (sensitivity $2 \mathrm{mV} /$ degree/sec) at a sampling rate of $102,4 \mathrm{~Hz}$. Sensor signals were transmitted to a tablet computer via Bluetooth ${ }^{\circledR}$ and stored for subsequent data analysis $[24,25]$. Machine learning algorithms were applied to calculate spatio-temporal gait parameters as mean per stride values derived from $4 \times 10 \mathrm{~m}$ gait tests (e.g. stride length, gait velocity) $[21,23]$. Gait variability as a measure of stride-to-stride variance is presented as Coefficient of 
Variance (CV) of each parameter using an average of 49 strides at HOSPITAL and 34 strides at HOME. Maximum toe clearance is the maximum distance between toe and ground at the end of the swing phase (prior to heel strike) as described in [26].

\section{Statistical analysis}

Normality of data was tested by Shapiro-Wilk test and variance homogeneity by Levene test. Due to the fact that gait variability parameters were not normally distributed, non-parametric analysis was performed for all gait parameters in order to detect differences between the repeated measures at HOSPITAL and at HOME (Paired Wilcoxon test). Cohen's d is presented as measure of effect size. Correlation analysis was performed using Spearman's rank correlation $\left(\mathrm{r}_{\mathrm{Sp}}\right)$ in order to evaluate the associations of gait parameters and clinical scores between both conditions. Statistics of reliability were assessed for clinical scores (UPDRS-III, PIGD, TUG), gait parameters representing short steps and bradykinesia (Stride length, Gait velocity, Stance time, Swing time, Stride time) as well as shuffling of gait (HS angle, TO angle, max TC) using Intra-Class Correlation Coefficients (ICC 2, 1) (two-way mixed, absolute, single measures). ICC analysis was not performed for gait variability parameters due to the fact that differences in recorded strides (HOSPITAL: 49, HOME: 34 strides) and varying standard deviations would provide unbalanced datasets that may affect reliability analysis. In order to minimize the effect of multiple comparisons, significance level was adapted by Bonferroni correction for multiple testing and p-values $<0.004$ were considered as significantly different $(p=0.05 / 13$ gait parameters $=0.004)$. All statistical analyses were performed using SPSS software package version 24 (IBM Corp. Released 2016. IBM $^{\circledR}$ SPSS $^{\circledR}$ Statistics for Windows, Version 24.0.0.2, Armonk, NY, USA: IBM Corp.).

\section{Results}

For all participants, the standardized gait tests were applicable in the HOME condition. Freezing of gait was not present in any of the participants, neither in the hospital nor at home recordings. In 
contrast to the flat surface in the hospital, several uneven surfaces may have influenced the participants' gait in the home environment. Carpets, carpeted floors or door-steps may be challenges in the daily life of PD patients, as well as narrow areas due to door frames or furniture. Furthermore, gait bout length differed between hospital and home (HOSPITAL: $10.0 \pm 0.3 \mathrm{~m}$; HOME: $7.8 \pm 1.5 \mathrm{~m}$ [range 6-10 m]; p 0.000). Figure S1 (Supplement) illustrates the walkway in HOSPITAL condition and an example of a walkway at a patient's home.

\section{Comparison of clinical and functional scores between HOSPITAL and HOME}

Motor impairment of participants was rated by a trained examiner using the UPDRS-III in the hospital and at patient's home. Assessments were performed at the same time of the day and in stable ON medication. UPDRS-III was comparable between HOSPITAL (mean \pm SD: $21.3 \pm 7.6$ points $)$ and HOME condition $(22.6 \pm 7.2$ points; $\mathrm{p}=0.071)$ with a trend towards a more severe motor impairment at home. Strong correlations were observed for UPDRS-III ratings in the hospital and at patient's home $\left(\mathrm{r}_{\mathrm{Sp}}=0.918, \mathrm{p}<0.000\right)$ (Figure 1). The TUG test as functional measure did not reveal significant differences between both settings (HOSPITAL: $11.1 \pm 2.9$ s; HOME: $10.9 \pm$ $3.1 \mathrm{~s} ; \mathrm{p}=0.262)$. Correlation analysis showed a strong association for TUG in the hospital and at home $\left(\mathrm{r}_{\mathrm{Sp}}=0.906, \mathrm{p}<0.000\right)$. From a clinical viewpoint, the motor status and functional performance was comparable between both conditions.

\section{Evaluation of gait parameter differences between HOSPITAL and HOME}

Spatiotemporal gait parameters significantly differed between HOSPITAL and HOME condition

(Table 2). Stride length (delta $\Delta$ presented as mean \pm SD and $\Delta_{\mathrm{p}}$ as change in percentage: $\Delta=-9.2 \pm$ $\left.9.4 \mathrm{~cm} ; \Delta_{\mathrm{p}}=-7.3 \%\right)$ and gait velocity $\left(\Delta=-0.07 \pm 0.11 \mathrm{~m} / \mathrm{s}, \Delta_{\mathrm{p}}=-6.1 \%\right)$ significantly decreased in the HOME setting. Toe-off angle $\left(\Delta=-3.4 \pm 3.1^{\circ} ; \Delta_{\mathrm{p}}=-5.4 \%\right)$, heel strike angle $\left(\Delta=-1.0 \pm 1.7^{\circ}\right.$; $\left.\Delta_{\mathrm{p}}=-8.5 \%\right)$ and maximum toe clearance $\left(\Delta=-0.7 \pm 2.5 \mathrm{~cm} ; \Delta_{\mathrm{p}}=-8.8 \%\right)$ significantly reduced from hospital to home-environment as well. In contrast, stride time $\left(\Delta=-0.02 \pm 0.05 \mathrm{~s} ; \Delta_{\mathrm{p}}=-1.8\right.$ 
$\%)$, swing time $\left(\Delta=-0.23 \pm 0.89 \% ; \Delta_{\mathrm{p}}=-0.5 \%\right)$, and stance time $\left(\Delta=+0.23 \pm 0.89 \% ; \Delta_{\mathrm{p}}=+0.5\right.$ \%) did not substantially change between HOSPITAL and HOME.

Parameters representing gait variability mostly increased from HOSPITAL to HOME condition, however, solely the rise of stride length CV nearly reached significance level $\left(\Delta=+2.5 \pm 3.3 \%\right.$; $\Delta_{\mathrm{p}}$ $=+35.3 \%)$. Swing time CV $\left(\Delta=+0.3 \pm 0.9 \% ; \Delta_{\mathrm{p}}=+7.8 \%\right)$, stance time CV $(\Delta=+0.2 \pm 0.5 \%$; $\left.\Delta_{\mathrm{p}}=+6.6 \%\right)$, and gait velocity $\mathrm{CV}\left(\Delta=+1.9 \pm 3.8 \% ; \Delta_{\mathrm{p}}=+21.8 \%\right)$ increased in standardized gait test at HOME, whereas stride time CV slightly decreased $\left(\Delta=-0.1 \pm 1.5 \% ; \Delta_{\mathrm{p}}=-3.0 \%\right)$.

\section{Correlations of gait parameters between standardized gait tests at HOSPITAL and at HOME}

Correlation analysis revealed strong correlations between sensor-based gait parameters recorded in the hospital and home environment (stride length $\mathrm{r}_{\mathrm{Sp}}=0.854, \mathrm{p}<0.000$; gait velocity $\mathrm{r}_{\mathrm{Sp}}=0.914$, $\mathrm{p}<0.000 ;$ stride time $\mathrm{r}_{\mathrm{Sp}}=0.814, \mathrm{p}<0.000 ;$ swing time $\mathrm{r}_{\mathrm{Sp}}=0.750, \mathrm{p}<0.000 ;$ stance time $\mathrm{r}_{\mathrm{Sp}}=$ 0.750, $\mathrm{p}<0.000$; toe-off angle $\mathrm{r}_{\mathrm{Sp}}=0.908, \mathrm{p}<0.000$; heel-strike angle $\mathrm{r}_{\mathrm{Sp}}=0.875, \mathrm{p}<0.000 ; \max$. toe clearance $r_{S p}=0.910, p<0.000$. Figure 2 illustrates example scatter plots and Bland-Altman plots for stride length and gait velocity. Both parameters show strong correlations between individual values of gait measures at HOSPITAL and at HOME. The mean difference of stride length was $9.2 \mathrm{~cm}$ and of gait velocity $0.08 \mathrm{~m} / \mathrm{s}$ with substantial inter-individual variability (min/max; stride length: $+5.5 /-35.1 \mathrm{~cm}$; gait velocity: $+0.15 /-0.33 \mathrm{~m} / \mathrm{s}$ [negative values indicate shorter steps and slower at home, respectively]). With regard to intra-individual differences, we observed two patients with increased gait velocity at HOME compared to HOSPITAL. All other participants walked slower $(n=10)$ or at similar gait velocity $(n=8$, lower than mean difference). One patient decreased gait velocity substantially indicating that massive individual differences in gait parameters are possible, even though clinical ratings were stable. This specific case is presented in the supplement in detail (Supplement). Intra-individual differences of gait velocity between both settings are presented in Figure 3. 
In contrast to spatiotemporal gait parameters, gait variability parameters did not show strong correlations. For swing time CV solely, a moderate correlation was observed between both conditions $\left(\mathrm{r}_{\mathrm{Sp}}=0.514, \mathrm{p}=0.020\right)$. All other gait variability parameters showed weak or no associations between standardized gait tests at HOSPITAL and at HOME (stride time CV: $\mathrm{r}_{\mathrm{Sp}}=$ $0.203, \mathrm{p}=0.391 ;$ stance time $\mathrm{CV}: \mathrm{r}_{\mathrm{Sp}}=0.349, \mathrm{p}=0.132 ;$ stride length $\mathrm{CV}: \mathrm{r}_{\mathrm{Sp}}=-0.051, \mathrm{p}=0.830$; and gait velocity $\left.\mathrm{CV}: \mathrm{r}_{\mathrm{Sp}}=-0.230, \mathrm{p}=0.329\right)$.

\section{Intra-Class correlation analysis between HOSPITAL and HOME}

Reliability analysis was performed for clinical scores and gait measures except of parameters representing gait variability due to large differences of detected strides at HOSPITAL and at HOME which may affect the interpretation of the coefficient of variance. Intra-Class correlation coefficients were excellent for clinical measures (UPDRS-III, PIGD subscore) and functional scores (TUG). Similarly, gait parameters representing short steps and bradykinesia (stride length, gait velocity, stance time, swing time, stride time) as well as parameters representing shuffling of gait (heel strike angle, toe-off angle, maximum toe clearance) showed excellent ICC's (Table 3).

\section{Subgroup analysis investigating the impact of walkway length and width}

With respect to the differences in 1) walkway length and 2) width, a subgroup analysis has been performed due to evaluate the impact of these differences. 1) Walkway length at home $\leq 7.5 \mathrm{~m}$ (Group 1, $\mathrm{n}=10)$ vs. $\geq 8 \mathrm{~m}$ (Group 2, n=10)): Gait parameter differences between hospital and home were similar regardless of shorter or longer walkways in the home environment. Comparing these results with the hospital condition (Table 2), there is no meaningful difference between subgroups and the entire cohort. 2) Walkway width: it appears that PD patients with wider walkways at home ( $\geq 85 \mathrm{~cm}$, Group 1, $\mathrm{n}=8$ ) show similar gait velocity as in the hospital condition (no significant difference, in contrast to the entire cohort). In contrast, narrow walkways (narrowest part of a 
walkway $<85 \mathrm{~cm}$, Group 2, $\mathrm{n}=12$ ) resulted in a significant reduction of gait velocity at home (Supplementary material).

\begin{tabular}{|c|c|c|c|c|c|c|c|c|c|c|c|c|c|}
\hline & \multicolumn{5}{|c|}{ Gait parameters HOSPITAL } & \multicolumn{5}{|c|}{ Gait parameters HOME } & \multicolumn{2}{|c|}{$\begin{array}{c}\text { Group } \\
\text { comparison * }\end{array}$} & \multirow{2}{*}{$\begin{array}{c}\begin{array}{c}\text { Effect } \\
\text { size }\end{array} \\
\begin{array}{c}\text { Cohen's } \\
\text { d }\end{array}\end{array}$} \\
\hline & mean & \pm & SD & Min & Max & mean & \pm & SD & Min & Max & $\mathbf{p}$ & $\mathbf{Z}$ & \\
\hline \multicolumn{14}{|c|}{ SHORT STEPS AND BRADYKINESIA } \\
\hline Stride Length (cm) & 125.2 & \pm & 19.4 & 86.6 & 160.8 & 116.0 & \pm & 21.3 & 76.3 & 157.5 & 0.000 & -3.66 & 2.85 \\
\hline Gait Velocity (m/s) & 1.14 & \pm & 0.23 & 0.63 & 1.52 & 1.07 & \pm & 0.23 & 0.64 & 1.50 & 0.004 & -2.88 & 1.68 \\
\hline Stride Time (s) & 1.11 & \pm & 0.09 & 0.98 & 1.39 & 1.09 & \pm & 0.08 & 0.95 & 1.27 & 0.232 & -1.20 & 0.56 \\
\hline Swing Time (\%) & 35.8 & \pm & 1.68 & 31.0 & 38.0 & 35.6 & \pm & 1.60 & 31.5 & 38.7 & 0.370 & -0.90 & 0.41 \\
\hline Stance Time (\%) & 64.2 & \pm & 1.68 & 62.0 & 69.0 & 64.4 & \pm & 1.60 & 61.3 & 68.5 & 0.370 & -0.90 & 0.41 \\
\hline \multicolumn{14}{|l|}{ SHUFFLING OF GAIT } \\
\hline TO Angle $\left({ }^{\circ}\right)$ & -64.5 & \pm & 7.37 & -78.2 & -51.0 & -61.0 & \pm & 8.60 & -76.5 & -43.1 & 0.000 & -3.73 & 3.02 \\
\hline HS Angle $\left(^{\circ}\right)$ & 11.8 & \pm & 4.39 & 2.8 & 21.9 & 10.8 & \pm & 5.26 & 0.5 & 22.9 & 0.000 & -3.92 & 3.64 \\
\hline $\max \mathrm{TC}(\mathrm{cm})$ & 8.0 & \pm & 3.15 & 3.5 & 14.1 & 7.3 & \pm & 4.42 & 3.0 & 21.2 & 0.000 & -2.61 & 1.44 \\
\hline \multicolumn{14}{|c|}{ REGULARITY OF GAIT } \\
\hline Stride Length CV (\%) & 7.08 & \pm & 1.46 & 4.72 & 9.74 & 9.58 & \pm & 2.99 & 5.02 & 16.85 & 0.005 & -2.80 & 1.61 \\
\hline Stride Time CV (\%) & 3.68 & \pm & 1.67 & 2.14 & 9.90 & 3.57 & \pm & 0.92 & 2.20 & 5.21 & 0.709 & -0.37 & 0.17 \\
\hline Swing Time CV (\%) & 4.36 & \pm & 1.38 & 2.63 & 7.92 & 4.70 & \pm & 1.52 & 2.64 & 8.87 & 0.086 & -1.72 & 0.83 \\
\hline Stance Time CV (\%) & 2.42 & \pm & 0.72 & 1.48 & 4.61 & 2.58 & \pm & 0.82 & 1.54 & 5.21 & 0.145 & -1.46 & 0.69 \\
\hline Gait Velocity CV (\%) & 8.55 & \pm & 2.10 & 6.07 & 14.75 & 10.41 & \pm & 2.80 & 5.31 & 17.16 & 0.052 & -1.94 & 0.96 \\
\hline
\end{tabular}

Table 2: Spatiotemporal gait parameters in HOSPITAL and HOME condition of PD patients $(n=20)$. Gait parameters representing gait variability are presented as coefficient of variance (CV). * Paired Wilcoxon-Test, TO Angle: toe off angle, HS Angle: heel strike angle, max TC: maximum toe clearance, Significance level p $<0.004$ (adapted), Bold numbers indicate significance. Gait parameters are categorized by gait characteristics defined by Gaßner et al. (2019) [8]; Schlachetzki et al. (2017) [6] 


\begin{tabular}{lcccc}
\hline CLINICAL SCORES & ICC $_{2,1}$ & $\mathbf{9 5} \% \mathbf{C l}$ & p-value & Cronbach's alpha \\
\hline UPDRS-III & 0.950 & $0.87-0.98$ & $<0.000$ & 0.955 \\
PIGD & 0.954 & $0.89-0.98$ & $<0.000$ & 0.953 \\
TUG & 0.968 & $0.92-0.99$ & $<0.000$ & 0.968 \\
\hline SHORT STEPS AND BRADYKINESIA & & & \\
\hline Stride length & 0.898 & $0.40-0.97$ & $<0.000$ & 0.944 \\
Gait velocity & 0.914 & $0.67-0.97$ & $<0.000$ & 0.940 \\
Stance time & 0.922 & $0.81-0-97$ & $<0.000$ & 0.922 \\
Swing time & 0.922 & $0.81-0.97$ & $<0.000$ & 0.922 \\
Stride time & 0.907 & $0.77-0.96$ & $<0.000$ & 0.912 \\
\hline SHUFFLING OF GAIT & & & & \\
\hline HS angle & 0.958 & $0.86-0.99$ & $<0.000$ & 0.967 \\
TO angle & 0.916 & $0.32-0.98$ & $<0.000$ & 0.960 \\
max TC & 0.907 & $0.77-0.96$ & $<0.000$ & 0.911 \\
\hline
\end{tabular}

Table 3: Reliability analyses comparing spatiotemporal gait parameters at HOSPITAL and at HOME using Intra-Class Correlation Coefficient analyses (two-way mixed, absolute, single measure). ICC: Intra-Class Correlation Coefficient; UPDRS-III: Motor score of the Unified Parkinson's disease rating scale; PIGD: Postural Instability and Gait Difficulty subscore; TUG: Timed Up and Go Test; HS angle: heel strike angle; TO Angle: toe off angle; max TC: maximum toe clearance. Significance level $\mathrm{p}<0.004$ (adapted), Bold numbers indicate significance. 


\section{Figure captions}
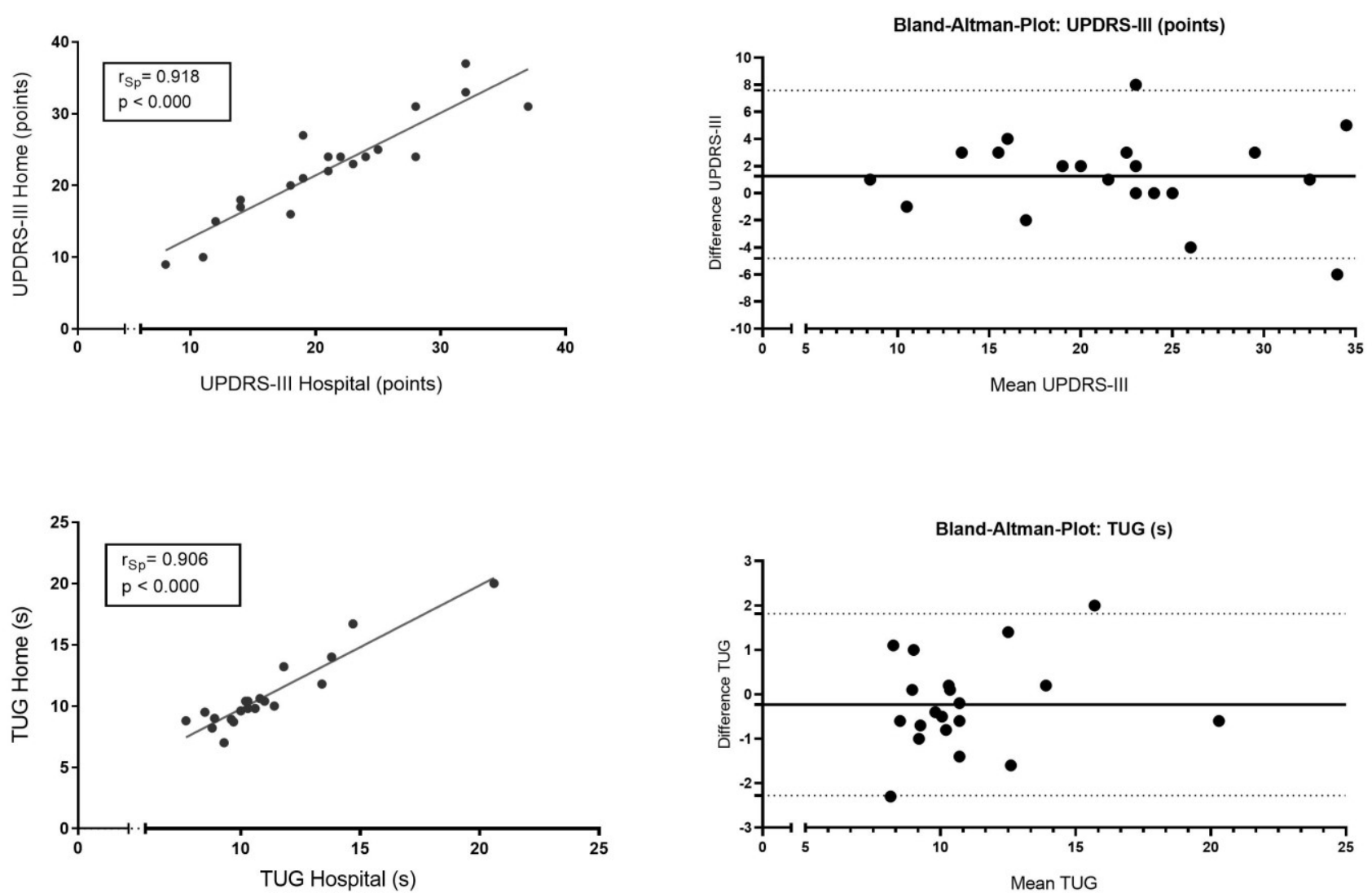

Figure 1: Correlation analysis and Bland-Altman Plots for the clinical rating of motor impairment using UPDRS-III and the functional motor performance using Timed Up and Go Test (TUG) between standardized assessments in the hospital (HOSPITAL) and home environment (HOME). UPDRS-III: Motor Score of the Unified Parkinson's Disease Rating Scale. $\mathrm{r}_{\mathrm{Sp}}$ : Spearman's rank correlation coefficient, Significance level $\mathrm{p}<0.004$ (adapted). 

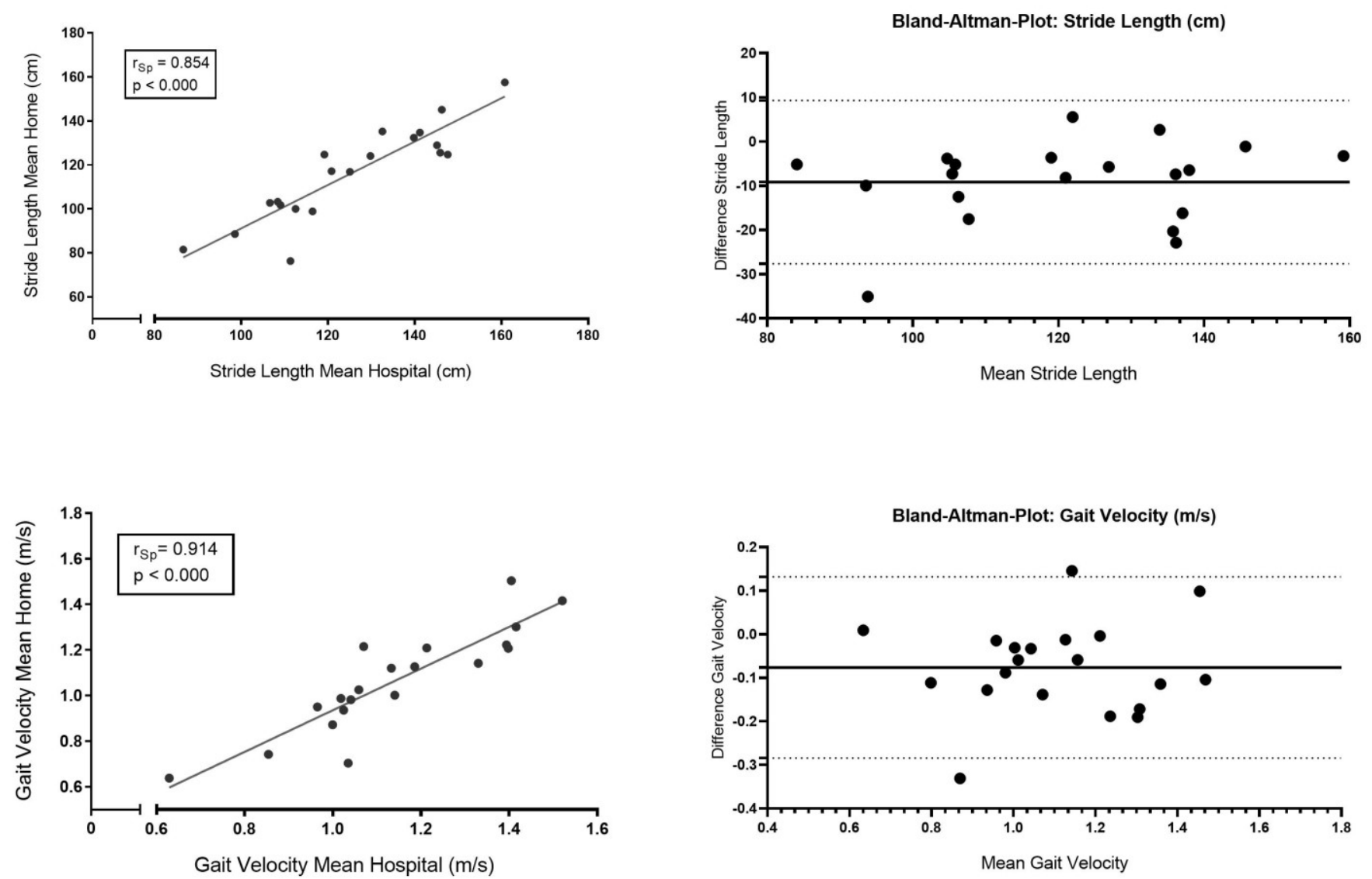

Figure 2: Left: Scatter plots and Bland-Altman Plots illustrating the relationship between sensor-based gait parameters (stride length, gait velocity) in the hospital (hospital) and home environment (home). $\mathrm{r}_{\mathrm{Sp}}$ : Spearman's rank correlation coefficient, Significance level p<0.004 (adapted). 


\section{Gait Velocity at Hospital and at Home}

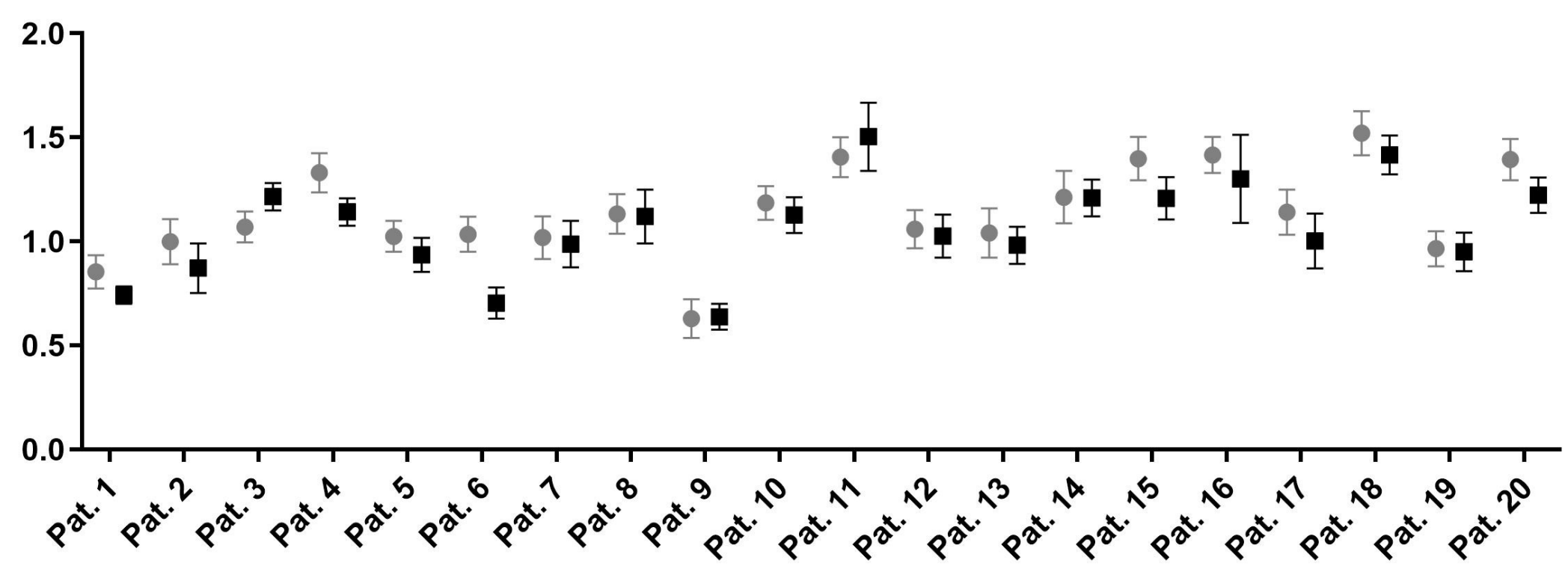

- Gait Velocity Hospital

- Gait Velocity Home

Figure 3: Intra-individual differences of gait velocity between standardized and supervised gait tests at HOSPITAL and at HOME 


\section{Discussion}

The aim of this pilot study was to evaluate the reliability of gait parameters derived from gait tests performed in hospital and home-environment in order to detect the clinical relevance of home-based gait measures. The main finding of this study was that PD patients' gait at HOME was slower, strides were shorter and shuffling was more present compared to the gait tests at HOSPITAL, even though clinical ratings were comparable. Interestingly, gait parameters reflecting bradykinesia, short steps and shuffling of gait show excellent reliability between both conditions.

\section{Clinical relevance of standardized gait measures at HOME}

This study investigated whether identical standardized, supervised gait tests performed at HOME are comparable to the test setting at HOSPITAL in order to evaluate the impact of environmental factors and detect the clinical relevance of home-based gait outcomes. The variance of spatiotemporal gait parameters were in the same range as the clinically established UPDRS-III as a similar pattern in the Bland-Altman plots demonstrated. Both, clinical gold standard (UPDRS-III) and sensor-based gait parameters representing short steps, bradykinesia, and shuffling of gait showed excellent Intra-Class correlation coefficients indicating that parameters assessed in the hospital and in the home-environment are reliable. However, significant differences in the absolute values of gait parameters at HOSPITAL and at HOME were observed. This finding indicates that gait parameters recorded in the home environment may not be directly compared to gait outcomes gained in the hospital. Nevertheless, within the cohort the picture of motor restrictions remained stable meaning that gait impairment shifted to lower values in most participants regardless of presenting impaired or unimpaired gait in the hospital. Even though gait parameters cannot be directly compared, they reflect gait impairment properly within the cohort. With regard to our fourstep-approach described in the introduction, standardized gait tests at HOME provide a reliable measure in comparison to gait measures in the hospital and may be used as an ideal bridge to close the gap between gait assessments in the HOSPITAL and continuous free-living gait. Herewith, we 
confirm that mobile health technologies complement rater-dependent clinical scores and may provide high quality information for patients' treatment [27] by their objective, metric, and quantitative character as well as by applicability in the home environment.

\section{Sensor-based gait parameters derived from free-living home-monitoring scenarios}

In our study, we observed excellent reliability for sensor-based gait parameters between standardized gait tests at HOSPITAL and at HOME condition. This is in contrast to studies which evaluated the comparability of in-lab and free-living environment. Ancum et al. compared a supervised and standardized gait test with free living gait in 254 older adults. They observed that gait speed recorded by the 4-m gait test is only weakly related to daily-life gait speed obtained from tri-axial lower-back accelerometer data over seven consecutive days [28]. Hillel et al. investigated a cohort of 150 elderly fallers and reported that in-lab values ( 1 min walk on a $15 \mathrm{~m}$ long corridor) for stride length, gait speed, step time as well as step and stride regularity do not reliably reflect the same parameters derived from 30s walking bouts during daily-walking [29]. In PD patients, especially gait parameters derived from longer free-living walking bouts ( $>30 \mathrm{~s})$ appeared to be clinically relevant since they differentiated between PD patients and controls [17]. From a technical point of view, it has recently been shown that detection of gait sequences in long-term homemonitoring of PD patients is possible, reliable, and efficient with an unsupervised algorithm [30]. These findings indicate that algorithms are able to automatically detect strides from free-living gait. However, gait outcomes derived from HOSPITAL and free-living conditions cannot easily be compared without considering context information or environmental factors.

\section{Comparability of gait outcomes at HOSPITAL and at HOME: Effect of Environmental factors}

Despite excellent reliability of gait parameters between both standardized conditions, we observed a significant decrease of stride length, gait velocity, heel strike angle, toe-off angle, and maximum toe-clearance in the at HOME setting. These differences may reflect the influence of environmental 
factors as uneven floor at home due to door steps and carpets or narrow areas due to door frames and furniture. Obviously, there was a substantial difference of the environments in the present study, among others there was a decrease of walkway length and width at HOME. It has been previously shown that walking environment has a significant effect on gait patterns. Patterson et al., investigated ten young adults over 30 minutes free-living gait in different walking conditions (flat/rough surface, on a hill, busy hallway) and reported significant differences in stride time [31]. Environmental factors also include the context of use since this information "going for a walk in the park" or "rushing to reach the bus" may be substantial for the interpretation of free-living gait patterns. In addition, there is a strong need for personalized models in order to cover individual confounders which can hugely vary between patients. In multiple sclerosis, a personalized algorithm solely predicted the maximum sustained gait speed of continuous walking with a sensor at home by the gait speed of the timed 25-foot-walk performed in the hospital with high accuracy [32]. This finding confirms the high relevance for personalized disease management which has recently been underlined for PD patients [33]. In addition, mobility measures are required to be defined for each disease separately [34]. Non-motor symptoms as mood and depression - not covered in the present study - should be included in personalized models as well since they may substantially affect gait patterns.

\section{Limitations}

A comparison between data of PD patients and controls was not performed in this pilot study that focused on the reliability and clinical relevance of sensor-based gait measures in PD patients at home. Future studies should include an age- and gender-matched control group in order to understand differences between both conditions (HOSPITAL and HOME) in more depth. A convenient sample was selected and no a-priori sample size calculation was conducted. Since it was the goal to evaluate the reliability of different test settings, HOME gait test was not matched for total walkway distance with the HOSPITAL setting. There may have been an order effect since 
HOME condition was always after testing in the HOSPITAL making the patients familiar with the protocol.

\section{Conclusion}

In conclusion, this pilot study presented that gait parameters reflecting bradykinesia, short steps and shuffling of gait show excellent reliability between HOSPITAL and HOME condition. However, PD patients' gait under standardized and supervised test conditions at HOME was slower, strides were shorter, and shuffling of gait was more present compared to the gait tests at HOSPITAL. Particularly, narrow walkways $(<85 \mathrm{~cm})$ resulted in a significant reduction of gait velocity at home. Consistent with our hypothesis, gait outcomes recorded in standardized tests at HOME are closely related to those captured at HOSPITAL but cannot be directly compared. They can be set as a clinically relevant reference for home-based recordings and may be the link between standardized gait tests in the hospital and free-living, continuous gait in everyday life. In future studies, standardized, supervised gait outcomes should be compared with non-standardized, non-supervised gait recordings. Potential confounders as fear of falling or falls, cognition and depression need to be evaluated. Furthermore, intra-individual and environmental factors including context information should be part of future studies investigating gait patterns outside the hospital. 


\section{Authors roles:}

Conception and design of the study: HG, PS, JK

Execution: HG, PS, FM, JW, JK

Statistical analysis: $\mathrm{HG}, \mathrm{PS}, \mathrm{AD}$

Writing the first draft of the manuscript: $\mathrm{HG}, \mathrm{AD}$

Manuscript review and critique: PS, FM, BE, JW, JK

\section{Ethical Standards}

This study has been approved by the local ethics committee in Erlangen, Germany and has therefore been performed in accordance with the ethical standards laid down in the 1964 Declaration of Helsinki and its later amendments.

\section{Conflicts of interest}

PS declares no conflict of interest.

HG, BE, and JK are supported by Mobilise-D from the Innovative Medicines Initiative 2 Joint Undertaking under grant agreement no. 820820. They further received an institutional research grant from the Bavarian Ministry of Economic Affairs and Media, Energy and Technology, Germany (Medical Valley Award 2016, Fall Risk PD).

HG and JW received an institutional research grant by the Federal Ministry of Education and Research (project: treatHSP, grant number 01GM1905). HG further received support by the Manfred Roth-Stiftung, Forschungsstiftung Medizin am Universitätsklinikum Erlangen, and the Förderverein für HSP-Forschung e.V.

HG and FM received an institutional research grant by the Huntington-Stiftung of the Deutsche Huntington Hilfe e.V.

FM is supported by the Interdisciplinary Center for Clinical Research of the FAU, Clinician Scientist programme.

BE holds ownerships of Portabiles HealthCare Technologies $\mathrm{GmbH}$ and Portabiles $\mathrm{GmbH}$, received compensation and honoraria from serving on scientific advisory boards for Abbvie $\mathrm{GmbH}$, Adidas $\mathrm{GmbH}$, Bosch Sensortec GmbH, and ST Sportservice GmbH. Further, he gratefully acknowledges the support of the German Research Foundation (DFG) within the framework of the Heisenberg professorship programme (grant number ES 434/8-1).

JW reports personal fees outside of the submitted work from Desitin Arzneimittel GmbH and Biogen GmbH.

JK received an Attract fellow grant (Digital Health Pathways in PD) by the Fraunhofer Gesellschaft. JK holds ownerships of Portabiles HealthCare Technologies GmbH and Portabiles $\mathrm{GmbH}$, received compensation and honoraria from serving on scientific advisory boards for LicherMT GmbH, Abbvie GmbH, UCB Pharma GmbH, GlaxoSmithKline GmbH \& Co. KG, Athenion $\mathrm{GmbH}$, and Thomashilfen $\mathrm{GmbH}$; as well as lecturing from UCB Pharma GmbH, TEVA Pharma GmbH, Licher MT GmbH, Desitin GmbH, Abbvie GmbH, Solvay Pharmaceuticals, and Ever Neuro Pharma GmbH. 


\section{References}

[1] Mirelman A, Bonato P, Camicioli R, Ellis TD, Giladi N, Hamilton JL, Hass CJ, Hausdorff JM, Pelosin E, Almeida QJ (2019) Gait impairments in Parkinson's disease. Lancet Neurol 18, 697-708.

[2] Baker JM (2018) Gait Disorders. Am J Med 131, 602-607.

[3] Debu B, De Oliveira Godeiro C, Lino JC, Moro E (2018) Managing Gait, Balance, and Posture in Parkinson's Disease. Curr Neurol Neurosci Rep 18, 23.

[4] Klucken J, Barth J, Kugler P, Schlachetzki J, Henze T, Marxreiter F, Kohl Z, Steidl R, Hornegger J, Eskofier B, Winkler J (2013) Unbiased and Mobile Gait Analysis Detects Motor Impairment in Parkinson's Disease. PLOS One 8, e56956.

[5] Espay AJ, Bonato P, Nahab F, Maetzler W, Dean JM, Klucken J, Eskofier BM, al. e (2016) Technology in Parkinson's Disease: Challenges and Opportunities. Mov Disord.

[6] Schlachetzki JCM, Barth J, Marxreiter F, Goßler J, Kohl Z, Reinfelder S, Gaßner H, Aminian K, Eskofier BM, Winkler J, Klucken J (2017) Wearable sensors objectively measure gait parameters in Parkinson's disease. PLOS One 10, e0183989.

[7] Marxreiter F, Gassner H, Borozdina O, Barth J, Kohl Z, Schlachetzki JCM, ThunHohenstein C, Volc D, Eskofier BM, Winkler J, Klucken J (2018) Sensor-based gait analysis of individualized improvement during apomorphine titration in Parkinson's disease. J Neurol 265, 2656-2665.

[8] Gassner H, Raccagni C, Eskofier BM, Klucken J, Wenning GK (2019) The Diagnostic Scope of Sensor-Based Gait Analysis in Atypical Parkinsonism: Further Observations. Front Neurol 10, 5.

[9] Flachenecker F, Gassner H, Hannik J, Lee DH, Flachenecker P, Winkler J, Eskofier B, Linker RA, Klucken J (2019) Objective sensor-based gait measures reflect motor impairment in multiple sclerosis patients: Reliability and clinical validation of a wearable sensor device. Mult Scler Relat Disord 39, 101903.

[10] Gaßner H, Jensen D, Marxreiter F, Kletsch A, Bohlen S, Schubert R, Muratori LM, Eskofier B, Klucken J, Winkler J, Reilmann R, Kohl Z (2020) Gait variability as digital biomarker of disease severity in Huntington's disease. Journal of neurology, 10.1007/s00415-0002009725-00413.

[11] Del Din S, Godfrey A, Mazza C, Lord S, Rochester L (2016) Free-living monitoring of Parkinson's disease: Lessons from the field. Mov Disord 31, 1293-1313.

[12] Eskofier BM, Lee SI, Baron M, Simon A, Martindale CF, Gassner H, Klucken J (2017) An Overview of Smart Shoes in the Internet of Health Things: Gait and Mobility Assessment in Health Promotion and Disease Monitoring. Applied Sciences-Basel 7.

[13] Pasluosta CF, Gassner H, Winkler J, klucken J, eskofier B (2015) An Emerging Era in the Management of Parkinson's Disease: Wearable Technologies and the Internet of Things. IEEE JOURNAL OF BIOMEDICAL AND HEALTH INFORMATICS 19, 1873-1881.

[14] Maetzler W, Klucken J, Horne M (2016) A clinical view on the development of technologybased tools in managing Parkinson's disease. Movement Disorders 31, 1263-1271.

[15] Catherine Morgan MR, Roisin McNaney, Bennet Jones, Lynn Rochester, Walter Maetzler, Ian Craddock, Alan L Whone (2020) Systematic Review Looking at the Use of Technology to Measure Free-Living Symptom and Activity Outcomes in Parkinson's Disease in the Home or a Home-like Environment. J Parkinsons Dis 10, 429-454.

[16] Morris R, Hickey A, Del Din S, Godfrey A, Lord S, Rochester L (2017) A model of freeliving gait: A factor analysis in Parkinson's disease. Gait Posture 52, 68-71.

[17] Del Din S, Godfrey A, Galna B, Lord S, Rochester L (2016) Free-living gait characteristics in ageing and Parkinson's disease: impact of environment and ambulatory bout length. $J$ Neuroeng Rehabil 13, 46.

[18] Robles-García V, Corral-Bergantiños Y, Espinosa N, Jácome MA, García-Sancho C, Cudeiro J, Arias P (2015) Spatiotemporal Gait Patterns During Overt and Covert Evaluation 
in Patients With Parkinson's Disease and Healthy Subjects: Is There a Hawthorne Effect? J Appl Biomech 31, 189-194.

[19] Hughes AJ, Daniel SE, Kilford L, Lees AJ (1992) Accuracy of clinical diagnosis of idiopathic Parkinson's disease: a clinico-pathological study of 100 cases. J Neurol Neurosurg Psychiatry 55, 181-184.

[20] Curtze C, Nutt JG, Carlson-Kuhta P, Mancini M, Horak FB (2015) Levodopa Is a DoubleEdged Sword for Balance and Gait in People With Parkinson's Disease. Mov Disord 30, 1361-1370.

[21] Barth J, Oberndorfer C, Pasluosta C, Schulein S, Gassner H, Reinfelder S, Kugler P, Schuldhaus D, Winkler J, Klucken J, Eskofier BM (2015) Stride segmentation during free walk movements using multi-dimensional subsequence dynamic time warping on inertial sensor data. Sensors (Basel) 15, 6419-6440.

[22] Podsiadlo D, Richardson S (1991) The timed "Up \& Go": a test of basic functional mobility for frail elderly persons. $J$ Am Geriatr Soc 39, 142-148.

[23] Rampp A, Barth J, Schulein S, Gassmann KG, Klucken J, Eskofier BM (2015) Inertial sensor-based stride parameter calculation from gait sequences in geriatric patients. IEEE Trans Biomed Eng 62, 1089-1097.

[24] Klucken J, Barth J, Kugler P, Schlachetzki J, Henze T, Marxreiter F, Kohl Z, Steidl R, Hornegger J, Eskofier B, Winkler J (2013) Unbiased and mobile gait analysis detects motor impairment in Parkinson's disease. PLoS One 8, e56956.

[25] Gassner H, Marxreiter F, Steib S, Kohl Z, Schlachetzki JCM, Adler W, Eskofier BM, Pfeifer K, Winkler J, Klucken J (2017) Gait and Cognition in Parkinson's Disease: Cognitive Impairment Is Inadequately Reflected by Gait Performance during Dual Task. Frontiers in Neurology 8, 550.

[26] Raccagni C, Gassner H, Eschlboeck S, Boesch S, Krismer F, Seppi K, Poewe W, Eskofier BM, Winkler J, Wenning G, Klucken J (2018) Sensor-based gait analysis in atypical parkinsonian disorders. Brain Behav, e00977.

[27] Hansen C, Sanchez-Ferro A, Maetzler W (2018) How Mobile Health Technology and Electronic Health Records Will Change Care of Patients with Parkinson's Disease. $J$ Parkinsons Dis 8, S41-s45.

[28] Van Ancum JM, van Schooten KS, Jonkman NH, Huijben B, van Lummel RC, Meskers CGM, Maier AB, Pijnappels M (2019) Gait speed assessed by a 4-m walk test is not representative of daily-life gait speed in community-dwelling adults. Maturitas 121, 28-34.

[29] Hillel I, Gazit E, Nieuwboer A, Avanzino L, Rochester L, Cereatti A, Croce UD, Rikkert MO, Bloem BR, Pelosin E, Del Din S, Ginis P, Giladi N, Mirelman A, Hausdorff JM (2019) Is every-day walking in older adults more analogous to dual-task walking or to usual walking? Elucidating the gaps between gait performance in the lab and during 24/7 monitoring. Eur Rev Aging Phys Act 16, 6.

[30] Ullrich M, Hannink J, Gaßner H, Klucken J, Eskofier BM, Kluge F (2019) in 2019 IEEE EMBS International Conference on Biomedical \& Health Informatics (BHI), pp. 1-4.

[31] Patterson MR, Whelan D, Reginatto B, Caprani N, Walsh L, Smeaton AF, Inomata A, Caulfield B (2014) Does external walking environment affect gait patterns? Conf Proc IEEE Eng Med Biol Soc 2014, 2981-2984.

[32] Supratak A, Datta G, Gafson AR, Nicholas R, Guo Y, Matthews PM (2018) Remote Monitoring in the Home Validates Clinical Gait Measures for Multiple Sclerosis. Front Neurol 9, 561.

[33] Bloem BR, Marks WJ, Jr., Silva de Lima AL, Kuijf ML, van Laar T, Jacobs BPF, Verbeek MM, Helmich RC, van de Warrenburg BP, Evers LJW, intHout J, van de Zande T, Snyder TM, Kapur R, Meinders MJ (2019) The Personalized Parkinson Project: examining disease progression through broad biomarkers in early Parkinson's disease. BMC Neurol 19, 160. 
[34] Shah VV, McNames J, Mancini M, Carlson-Kuhta P, Spain RI, Nutt JG, El-Gohary M, Curtze C, Horak FB (2020) Quantity and quality of gait and turning in people with multiple sclerosis, Parkinson's disease and matched controls during daily living. J Neurol 267, 11881196. 


\section{Supplementary material}
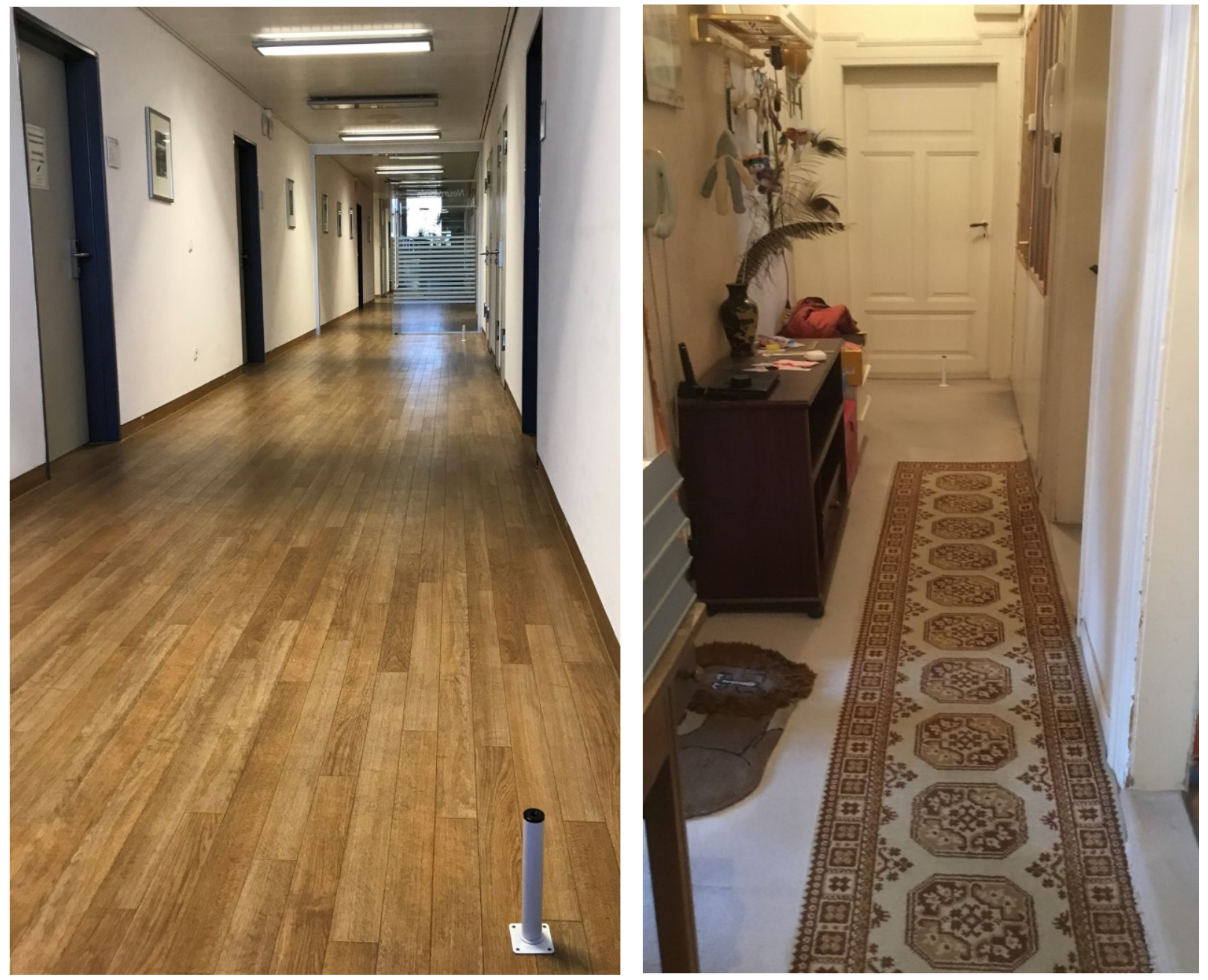

Figure S1: Left: Walkway in the hospital (length $10 \mathrm{~m}$, width $220 \mathrm{~cm}$ ), Right: Example of a walkway in one patient's home with a walkway length of $6 \mathrm{~m}$ and width of $80 \mathrm{~cm}$. 


\section{Case description}

In the following, the change of gait parameters between HOSPITAL and HOME is described for one study participant. This male PD patient, 73 years old with a disease duration of 6 years is marked in the figures using a red circle. He was tested in both conditions with comparable clinical motor symptoms as rated by the UPDRS-III and PIGD subscore. However, functional measures (TUG) increased and sensor-based gait parameters (e.g. stride length, gait velocity) substantially decreased in the home-environment (see Table S1, Figure S2).

\begin{tabular}{|l|l|l|}
\hline Pat. 6 & hospital & home \\
\hline UPDRS-III & 32 & 33 \\
\hline PIGD & 5 & 4 \\
\hline TUG & 14,7 & 16,7 \\
\hline Stride length [cm] & 111,4 & 76,3 \\
\hline Gait velocity [m/s] & 1,03 & 0,70 \\
\hline Walkway length [m] & 10 & $6($ see Figure S1) \\
\hline Walkway width $(\mathrm{cm})$ & 220 & 80 (see Figure S1) \\
\hline No cognitive impairment (MoCA = 28) \\
\hline Low fear of falling (FES-I = 19) \\
\hline No fall history
\end{tabular}

Table S1: Patient characteristics of Pat. 6
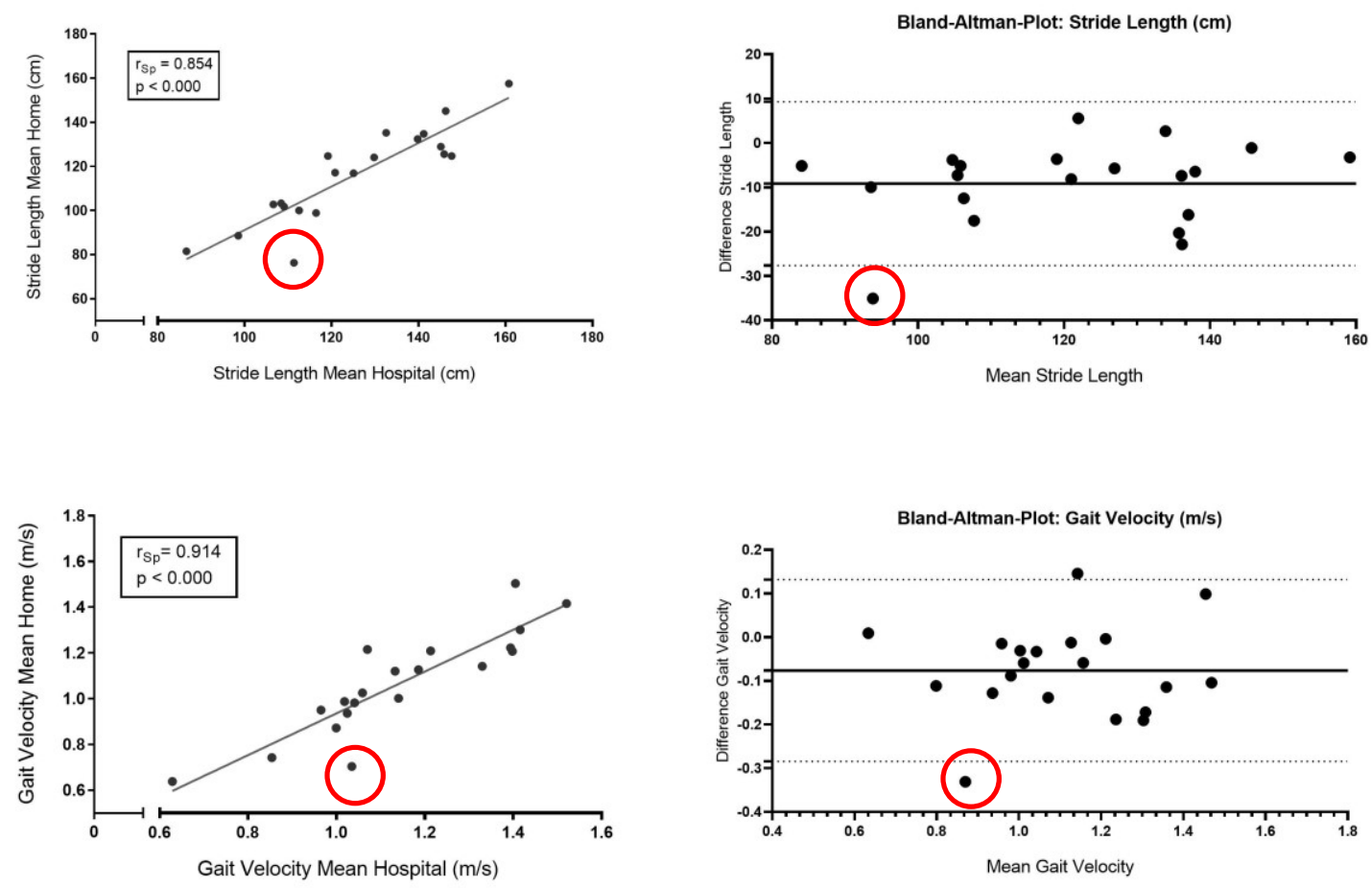

Figure S2: Stride length and gait velocity strongly dropped in Patient 6 (red circle). 


\section{Gait Velocity at Hospital and at Home}

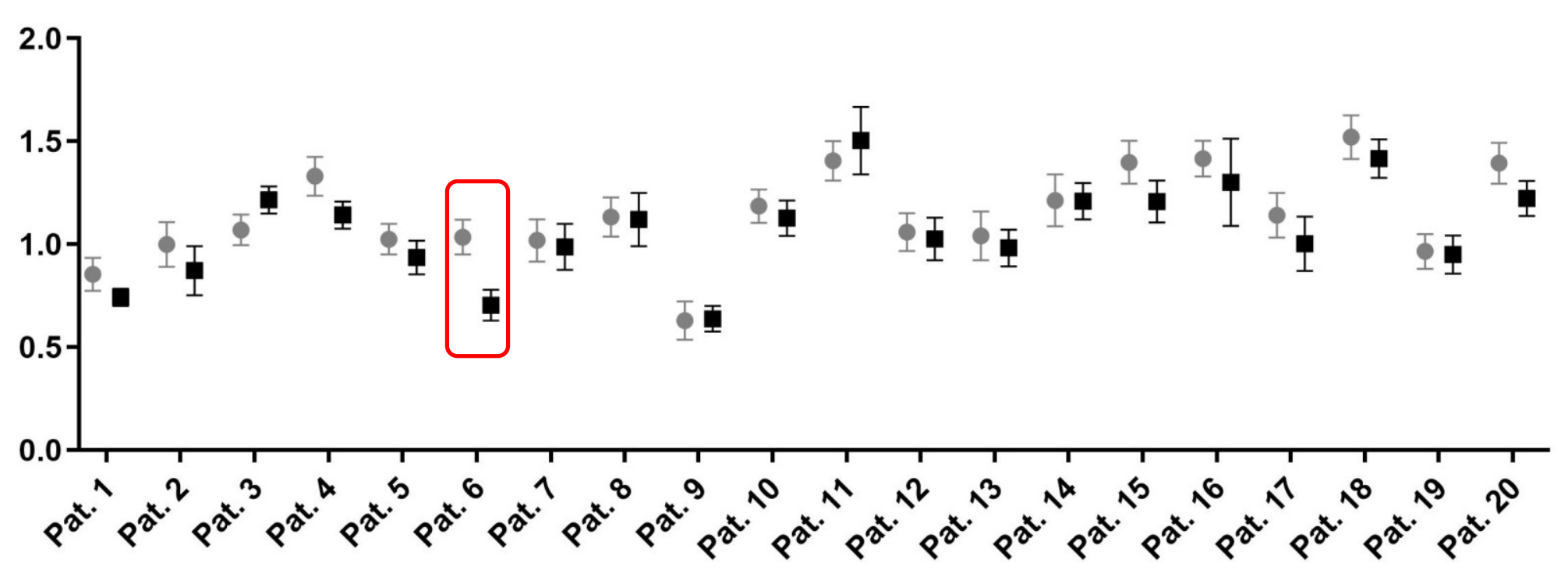

- Gait Velocity Hospital

- Gait Velocity Home

Figure S3: substantial drop of gait velocity between standardised gait tests in the hospital (HOSPITAL) and home environment (HOME).

\section{Conclusion:}

In contrast to the other 19 participants in this study, Pat. 6 showed a massive drop of gait outcomes without differences in clinical motor symptoms. Walkway length and width may have substantially influenced his gait measures at home. Motivational aspects as mood and depression could have played a major role but were not investigated in this pilot study. These measures should be covered in detail in future studies. 
Table S2: Subgroup analysis - walkway length: Differentiation between patients with walkway length at home $\leq 7.5 \mathrm{~m}($ Group 1, $\mathrm{n}=10)$ and $\geq 8 \mathrm{~m}($ Group 2, $\mathrm{n}=10)$

\begin{tabular}{|c|c|c|c|c|c|c|}
\hline Gait parameters Mean: Home-Gait bout & Mean & SD & Min & Max & $\mathbf{p}$ & $\mathbf{Z}$ \\
\hline Stride Length [cm] @Hospital, Group 1 & 118.4 & 18.6 & 86.6 & 147.6 & \multirow{2}{*}{0.009} & \multirow{2}{*}{-2.60} \\
\hline Stride Length [cm] @Home, Group 1 & 106.7 & 20.4 & 75.5 & 132.4 & & \\
\hline Stride Length [cm] @Hospital, Group 2 & 131.9 & 18.7 & 106.6 & 160.8 & \multirow{2}{*}{0.007} & \multirow{2}{*}{-2.70} \\
\hline Stride Length [cm] @Home, Group 2 & 124.0 & 18.0 & 99.9 & 152.0 & & \\
\hline Gait velocity [m/s] @Hospital, Group 1 & 1.03 & 0.19 & 0.63 & 1,33 & \multirow{2}{*}{0.059} & \multirow{2}{*}{-1.89} \\
\hline Gait velocity [m/s] @Home, Group 1 & 0.95 & 0.20 & 0.64 & 1.22 & & \\
\hline Gait velocity [m/s] @Hospital, Group 2 & 1.26 & 0.19 & 0.97 & 1.52 & \multirow{2}{*}{0.022} & \multirow{2}{*}{-2.29} \\
\hline Gait velocity [m/s] @Home, Group 2 & 1.18 & 0.19 & 0.95 & 1.50 & & \\
\hline Stride time [s] @Hospital, Group 1 & 1.16 & 0.09 & 1.07 & 1.39 & \multirow{2}{*}{0.059} & \multirow{2}{*}{-1.89} \\
\hline Stride time [s] @Home, Group 1 & 1.13 & 0.08 & 1.03 & 1.27 & & \\
\hline Stride time [s] @Hospital, Group 2 & 1.06 & 0.05 & 0.99 & 1.14 & \multirow{2}{*}{0.878} & \multirow{2}{*}{-0.15} \\
\hline Stride time [s] @Home, Group 2 & 1.05 & 0.06 & 0.95 & 1.14 & & \\
\hline Swing Time [\%] @ Hospital Group 1 & 35.3 & 1.93 & 31.0 & 37.3 & \multirow{2}{*}{0.575} & \multirow{2}{*}{-0.56} \\
\hline Swing Time [\%]@Home Group 1 & 35.0 & 1.54 & 31.5 & 37.0 & & \\
\hline Swing Time [\%] @ Hospital Group 2 & 36.3 & 1.33 & 33.7 & 38.0 & \multirow{2}{*}{0.575} & \multirow{2}{*}{-0.56} \\
\hline Swing Time [\%] @Home, Group 2 & 36.1 & 1.54 & 33.2 & 38.7 & & \\
\hline Stance Time [\%] @Hospital Group 1 & 64.7 & 1.93 & 62.7 & 69.0 & \multirow{2}{*}{0.575} & \multirow{2}{*}{-0.56} \\
\hline Stance Time [\%] @Home Group 1 & 65.0 & 1.54 & 63.0 & 68.6 & & \\
\hline Stance Time [\%] @ Hospital Group 2 & 63.7 & 1.34 & 62.0 & 66.3 & \multirow{2}{*}{0.575} & \multirow{2}{*}{-0.56} \\
\hline Stance Time [\%] @Home Group 2 & 63.9 & 1.54 & 61.3 & 66.8 & & \\
\hline TO angle $\left[{ }^{\circ}\right]$ @Hospital, Group 1 & $-63,2$ & 7,60 & $-71,8$ & $-53,1$ & \multirow{2}{*}{0,007} & \multirow{2}{*}{$-2,70$} \\
\hline TO angle $\left[{ }^{\circ}\right] @$ Home, Group 1 & $-59,1$ & 10,31 & $-69,7$ & $-43,1$ & & \\
\hline TO angle $\left[{ }^{\circ}\right]$ @Hospital. Group 2 & $-65,8$ & 7,28 & $-78,2$ & $-51,0$ & \multirow{2}{*}{0,009} & \multirow{2}{*}{$-2,60$} \\
\hline TOangle [] @Home, Group 2 & $-62,9$ & 6,43 & $-76,6$ & $-52,2$ & & \\
\hline HS angle [ $\left.{ }^{\circ}\right] @$ Hospital, Group 1 & 10,3 & 4,87 & 2,84 & 17,7 & \multirow{2}{*}{0,009} & \multirow{2}{*}{$-2,60$} \\
\hline HS angle $\left[^{\circ}\right] @$ Home, Group 1 & 8,86 & 5,41 &,- 46 & 17,0 & & \\
\hline HS angle $\left[{ }^{\circ}\right] @$ Hospital, Group 2 & 13,2 & 3,45 & 9,36 & 21,9 & \multirow{2}{*}{0,721} & \multirow{2}{*}{$-0,36$} \\
\hline HS angle $\left[^{\circ}\right] @$ Home, Group 2 & 12,7 & 4,59 & 5,92 & 22,9 & & \\
\hline Max TC [cm] @Hospital, Group 1 & 6,94 & 2,76 & 3,47 & 13,0 & \multirow{2}{*}{0,047} & -199 \\
\hline Max TC [cm] @Home, Group 1 & 5,98 & 3,61 & 2,96 & 15,2 & & \\
\hline Max TC [cm] @Hospital, Group 2 & 9,11 & 3,27 & 4,22 & 14,1 & 7 01 & 170 \\
\hline Max TC [cm] @Home, Group 2 & 8,66 & 4,92 & 3,28 & 21,2 & 0,014 & $-1,10$ \\
\hline
\end{tabular}

Group 1: @Home walkway length $\leq 7.5 \mathrm{~m}(\mathrm{n}=10)$

Group 2: @Home walkway length $\geq 8$ m $(n=10)$

With regard to walkway length, gait parameter differences between hospital and home were similar regardless of shorter or longer walkways in the home environment. Comparing these results with the hospital condition (Table 2 in the manuscript), there is no meaningful difference between subgroups and the entire cohort.

A comparison between patients with walkway length $=10 \mathrm{~m}$ as in the hospital condition and $<10 \mathrm{~m}$ did not provide additional information and need to be interpreted cautiously due to the small sample size $(n=4$ in the group with walkway length $=10 \mathrm{~m})$. In figure $\mathrm{S} 4$, results of a correlation analysis suggest very small correlations between gait parameter differences (hospital - home) and gait bout 
length meaning that e.g. gait velocity changed in the home environment independent of gait bout length.

Figure S4: Correlation analysis between delta gait velocity / delta stride length and differences of walkway length.
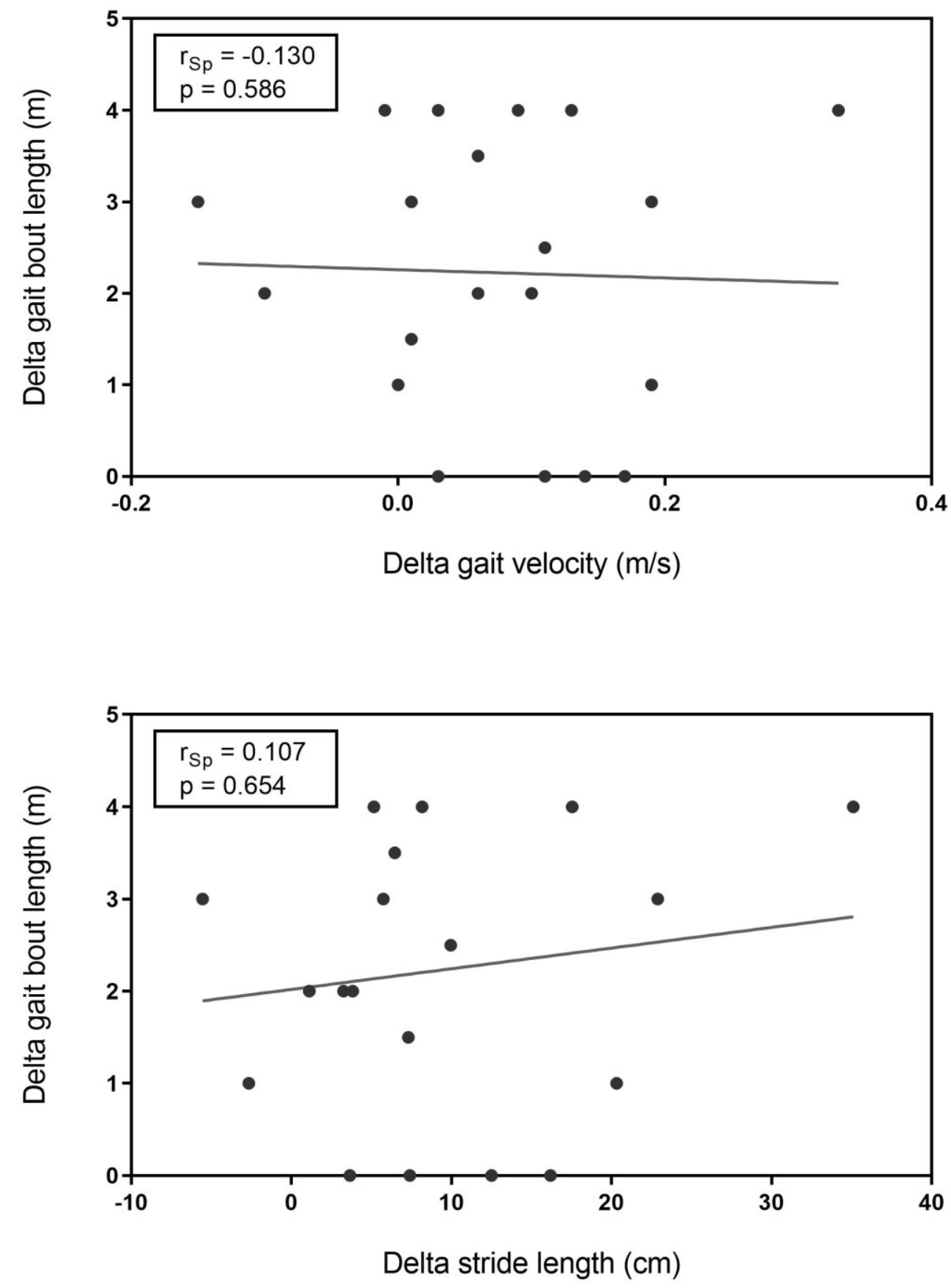
Table S3: Subgroup analysis - walkway width: Differentiation between wider $\geq 85 \mathrm{~cm}$

(Group 1, $\mathrm{n}=8$ ) and narrow $<85 \mathrm{~cm}$ (Group 2, $\mathrm{n}=12$ ) walkways at home. The walkway width describes the narrowest part of the walkway, e.g. if participants cross a door.

\begin{tabular}{|c|c|c|c|c|c|c|}
\hline Gait parameters Mean: Narrowness & Mean & SD & Min & Max & $\mathbf{p}$ & $\mathbf{Z}$ \\
\hline Stride Length [cm] @Hospital Group 1 & 125.2 & 17.9 & 98.5 & 146.2 & \multirow{2}{*}{0.036} & \multirow{2}{*}{-2.10} \\
\hline Stride Length [cm] @Home Group 1 & 117.8 & 18.7 & 88.6 & 142.1 & & \\
\hline Stride Length [cm] @ Hospital Group 2 & 125.2 & 21.1 & 86.6 & 160.8 & \multirow{2}{*}{0.003} & \multirow{2}{*}{-2.98} \\
\hline Stride Length [cm] @Home, Group 2 & 113.8 & 22.6 & 75.5 & 152.0 & & \\
\hline Gait velocity [m/s] @ Hospital Group 1 & 1.16 & 0.21 & 0.85 & 1.42 & \multirow[b]{2}{*}{0.263} & \multirow{2}{*}{-1.12} \\
\hline Gait velocity [m/s] @Home Group 1 & 1.12 & 0.25 & 0.74 & 1.50 & & \\
\hline Gait velocity [m/s] @ Hospital Group 2 & 1.13 & 0.23 & 0.63 & 1.52 & \multirow{2}{*}{0.004} & \multirow{2}{*}{-2.90} \\
\hline Gait velocity [m/s] @Home, Group 2 & 1.03 & 0.22 & 0.64 & 1.42 & & \\
\hline Stride time [s] @ Hospital Group 1 & 1.09 & 0.07 & 1.00 & 1.17 & \multirow{2}{*}{0.327} & \multirow{2}{*}{-0.98} \\
\hline Stride time [s] @Home, Group 1 & 1.07 & 0.08 & 0.95 & 1.19 & & \\
\hline Stride time [s] @ Hospital Group 2 & 1.13 & 0.11 & 0.99 & 1.39 & \multirow[b]{2}{*}{0.530} & \multirow[b]{2}{*}{-0.63} \\
\hline Stride time [s] @Home, Group 2 & 1.11 & 0.08 & 1.00 & 1.27 & & \\
\hline Swing Time [\%] @ Hospital Group 1 & 35.9 & 1.26 & 33.48 & 37.5 & \multirow{2}{*}{1.000} & \multirow{2}{*}{0.00} \\
\hline Swing Time [\%]@Home Group 1 & 35.9 & 1.41 & 34.17 & 38.70 & & \\
\hline Swing Time [\%] @ Hospital Group 2 & 35.7 & 1.96 & 30.97 & 38.0 & \multirow{2}{*}{0.239} & \multirow{2}{*}{-1.177} \\
\hline Swing Time [\%] @Home, Group 2 & 35.4 & 1.74 & 31.45 & 37.7 & & \\
\hline Stance Time [\%] @Hospital Group 1 & 64.1 & 1.26 & 62.50 & 66.5 & \multirow{2}{*}{1.000} & \multirow{2}{*}{0.00} \\
\hline Stance Time [\%] @Home Group 1 & 64.1 & 1.41 & 61.31 & 65.8 & & \\
\hline Stance Time [\%] @ Hospital Group 2 & 64.3 & 1.96 & 62.00 & 69.0 & \multirow{2}{*}{0.239} & \multirow{2}{*}{-1.177} \\
\hline Stance Time [\%] @Home Group 2 & 64.6 & 1.74 & 62.35 & 68.6 & & \\
\hline TO angle $\left[{ }^{\circ}\right] @$ Hospital, Group 1 & $-64,3$ & 9.18 & -78.24 & $-51,0$ & \multirow{2}{*}{0,069} & \multirow{2}{*}{$-1,82$} \\
\hline TO angle $\left[^{\circ}\right] @$ Home, Group 1 & $-62,3$ & 9,22 & $-76,55$ & $-48,0$ & & \\
\hline TO angle $\left[{ }^{\circ}\right]$ @Hospital. Group 2 & $-64,6$ & 6,33 & $-71,89$ & $-53,1$ & \multirow{2}{*}{0,002} & \multirow{2}{*}{$-3,06$} \\
\hline TOangle [] @Home, Group 2 & $-60,1$ & 8,46 & $-69,69$ & $-43,1$ & & \\
\hline HS angle [ $\left.{ }^{\circ}\right]$ @Hospital, Group 1 & 11,1 & 6,01 & 2,84 & 21,9 & \multirow{2}{*}{0,401} & \multirow{2}{*}{$-0,84$} \\
\hline HS angle [ $\left.{ }^{\circ}\right] @$ Home, Group 1 & 10,5 & 7,06 & $-0,46$ & 22,9 & & \\
\hline HS angle $\left[{ }^{\circ}\right] @$ Hospital, Group 2 & 12,2 & 3,11 & 6,36 & 17,7 & \multirow[b]{2}{*}{0,005} & \multirow[b]{2}{*}{$-1,96$} \\
\hline HS angle $\left[^{\circ}\right]$ @Home, Group 2 & 11,0 & 3,99 & 3,12 & 17,0 & & \\
\hline Max TC [cm] @Hospital, Group 1 & 8,67 & 3,49 & 5,38 & 14,1 & \multirow{2}{*}{0,161} & \\
\hline Max TC [cm] @Home, Group 1 & 8,25 & 5,75 & 2,96 & 21,2 & & $-1,40$ \\
\hline Max TC [cm] @Hospital, Group 2 & 7,59 & 2,97 & 3,47 & 13,0 & & \\
\hline Max TC [cm] @Home, Group 2 & 6,69 & 3,40 & 3,28 & 15,2 & 0,023 & $-2,27$ \\
\hline
\end{tabular}

Group 1: @Home narrow point $\geq 85 \mathrm{~cm}(\mathrm{~N}=8)$

Group 2: @Home narrow point < $85 \mathrm{~cm}(\mathrm{~N}=12)$

With regard to narrowness, it appears that PD patients with wider walkways at home ( $\geq 85 \mathrm{~cm}$, Group 1) show similar gait velocity as in the hospital condition (no significant difference, in contrast to the entire cohort, marked in red). Whereas narrow walkways $(<85 \mathrm{~cm}$ (Group 2) resulted in a significant reduction of gait velocity at home. We chose the cut-off point at $85 \mathrm{~cm}$ since this is in the range of the standardized width of a German doorframe. 
Table S4: Subgroup analysis - cognition: Differentiation between patients without (Group 1: $\mathrm{MoCa} \geq 26, \mathrm{n}=15$ ) and with (Group 2: $\mathrm{MoCa}<26, \mathrm{n}=5$ ) cognitive impairment.

\begin{tabular}{|c|c|c|c|}
\hline Gait parameters Mean: Cognition & Mean & SD & $\mathbf{p}$ \\
\hline Stride Length [cm] @Hospital Group 1 & 125.2 & 21.1 & \multirow{2}{*}{0.002} \\
\hline Stride Length [cm] @Home Group 1 & 115.5 & 22.8 & \\
\hline Stride Length [cm] @ Hospital Group 2 & 124.0 & 15.0 & \multirow{2}{*}{0.043} \\
\hline Stride Length [cm] @Home, Group 2 & 117.6 & 18.5 & \\
\hline Gait velocity [m/s] @ Hospital Group 1 & 1.15 & 0.24 & \multirow{2}{*}{0.008} \\
\hline Gait velocity [m/s] @Home Group 1 & 1.05 & 0.23 & \\
\hline Gait velocity [m/s] @ Hospital Group 2 & 1.12 & 0.16 & \multirow{2}{*}{0.345} \\
\hline Gait velocity [m/s] @Home, Group 2 & 1.10 & 0.24 & \\
\hline Stride time [s] @ Hospital Group 1 & 1.11 & 0.10 & \multirow{2}{*}{0.570} \\
\hline Stride time [s] @Home, Group 1 & 1.10 & 0.08 & \\
\hline Stride time [s] @ Hospital Group 2 & 1.11 & 0.07 & \multirow{2}{*}{0.138} \\
\hline Stride time [s] @Home, Group 2 & 1.08 & 0.08 & \\
\hline Swing Time [\%] @ Hospital Group 1 & 35.9 & 1.80 & \multirow{2}{*}{0.460} \\
\hline Swing Time [\%]@Home Group 1 & 35.6 & 1.49 & \\
\hline Swing Time [\%] @ Hospital Group 2 & 35.5 & 1.41 & \multirow{2}{*}{0.686} \\
\hline Swing Time [\%] @Home, Group 2 & 35.4 & 2.09 & \\
\hline Stance Time [\%] @Hospital Group 1 & 64.1 & 1.80 & \multirow{2}{*}{0.460} \\
\hline Stance Time [\%] @Home Group 1 & 64.4 & 1.49 & \\
\hline Stance Time [\%] @ Hospital Group 2 & 64.5 & 1.41 & \multirow{2}{*}{0.686} \\
\hline Stance Time [\%] @Home Group 2 & 64.6 & 2.09 & \\
\hline TO angle $\left[{ }^{\circ}\right] @$ Hospital, Group 1 & $-64,2$ & 7,41 & \multirow{2}{*}{0,001} \\
\hline TO angle $\left[{ }^{\circ}\right] @$ Home, Group 1 & $-61,1$ & 9,55 & \\
\hline TO angle $\left[{ }^{\circ}\right] @$ Hospital. Group 2 & -62.3 & 7.60 & \multirow{2}{*}{0,138} \\
\hline TOangle [] @Home, Group 2 & $-60,6$ & 5.62 & \\
\hline HS angle $\left[{ }^{\circ}\right] @$ Hospital, Group 1 & 12.2 & 4.36 & \multirow{2}{*}{0,125} \\
\hline HS angle $\left[{ }^{\circ}\right] @$ Home, Group 1 & 11.3 & 5.11 & \\
\hline HS angle [ $\left.{ }^{\circ}\right] @$ Hospital, Group 2 & 10.5 & 4.68 & \multirow{2}{*}{0,043} \\
\hline HS angle $\left[^{\circ}\right]$ @Home, Group 2 & 9.14 & 6.00 & \\
\hline Max TC [cm] @Hospital, Group 1 & 8.19 & 3.49 & \multirow{2}{*}{0,053} \\
\hline Max TC [cm] @Home, Group 1 & 7.76 & 4.93 & \\
\hline Max TC [cm] @Hospital, Group 2 & 7,54 & 1.98 & \multirow{2}{*}{0,043} \\
\hline Max TC [cm] @Home, Group 2 & 5.99 & 2.17 & \\
\hline
\end{tabular}

\section{Group 1: $\mathrm{MoCa} \geq 26(\mathrm{n}=15)$}

Group 2: $\mathrm{MoCa}<26(n=5)$

We figured out, that gait velocity and TO angle in patients without cognitive impairment showed a significant difference between hospital and home. In contrast, patients with MoCA $<26$ (mild cognitive impairment) didn't show these differences. However, as the data samples are rather small and not well balanced with respect to group size $(n=15$ vs. $n=5)$, these results should be interpreted very cautiously. 